\title{
MicroRNA-588 regulates invasion, migration and epithelial-mesenchymal transition via targeting EIF5A2 pathway in gastric cancer
}

This article was published in the following Dove Press journal: Cancer Management and Research

\author{
Xiaorong Zhou' \\ Manli Xu \\ Yonghong Guo' \\ Ling $\mathrm{Ye}^{1}$ \\ Limin Long' \\ Haiqin Wang' \\ Pan Tan' \\ Meili $\mathrm{Xu}^{\prime}$ \\ 'Department of Gerontology, The \\ Second Xiangya Hospital, Central \\ South University, Changsha, Hunan \\ 4I00II, China; ${ }^{2}$ Department of Basic \\ Medicine, Guizhou College of Health \\ Professions, Tongren, Guizhou, 554300, \\ China
}

Correspondence: Meili Xu Department of Gerontology, The Second Xiangya Hospital, Central South University, No 139 Middle Renmin Road, Changsha, Hunan 4100II, China Tel/fax +86 073I 85292429

Email doctormeili@csu.edu.cn
Background: miRNAs are potential regulators of genes in many cancers. Here, we confirmed that the expression of miR-588 decreased in gastric cancer (GC) tissues and cells.

Materials and methods: Sixty-seven GC tissues along with noncancerous tissues adjacent to them were included in the study. Quantitative real-time reverse transcription-PCR study was done to quantify the expression levels of mature miRNA. The expression of proteins was determined by Western blot and transwell chamber assay for invasion and migration studies. Immunohistochemical analysis and luciferase assay were done for evaluating the expression of epithelial-mesenchymal transition (EMT) markers and activity of EIF5A2, respectively. In vivo metastatic assay was done by injecting MGC-803 cells into nude mice.

Results: In the 5-year predicted survival study of GC patients included in the study, we found that miR-588 acted as a specific prognostic marker. Overexpression of miR-588 resulted in suppression of cell invasion, migration and progression of EMT, whereas suppression of miR588 inverted the effects in both in vivo and in vitro experiments. miR-588 retained EIF5A2 by directly binding to the 3'-UTR. EIF5A2 was overexpressed in GC tissue samples, and the expression of miR-588 was inversely correlated to the levels of EIF5A2. The impact of miR588 on invasion, migration and progression of EMT may be partially due to miR-588-mediated alterations of EiF5A2.

Conclusion: Overall, the findings of the study suggest that miR-588 acts as a tumor suppressor by regulating the invasion, migration and EMT via EIF5A2 pathway, hence presenting miR-588 as a prognostic marker as well as a therapeutic target for GC.

Keywords: miR-588, gastric cancer, EIF5A2, EMT

\section{Introduction}

Gastric cancer (GC) has been identified as a common malignancy globally and is found to have high recurrence rate with the feature of distant metastasis. ${ }^{1,2}$ Metastasis of GC occurs via multistep pathways called as the "invasion-metastasis cascade". These multistep pathways cause genetic and epigenetic alterations affecting the very important epithelial-mesenchymal transition (EMT) in cells. ${ }^{3}$ A remarkable feature of EMT is loss of homotypic adhesion and acquiring mesenchymal characters in cells, which is supported by various factors such as microRNAs, transcription factors and noncoding RNAs. ${ }^{4}$ Even with progress in methods of diagnosis and related therapies, the chances of endurance in GC remain bounded. ${ }^{5,6}$ Therefore, search of the possible regulatory mechanism responsible for metastasis of GC is very crucial for increasing the chances of survival.

MicroRNAs, also called as miRNAs, are a group of small noncoding RNAs. They have the property to regulate the expression of genes by altering the 3 '-UTR region of the 
specific genes leading to degradation of mRNA.${ }^{78}$ Literatures indicate miRNAs can induce carcinogenesis by inhibiting tumor suppressor genes and also can act as tumor suppressor by downregulating oncogenes. ${ }^{9}$ Reports have suggested such an uncommon behavior of miRNAs in many cancers including GC. ${ }^{10-15}$ miRNAs affect a number of cellular processes such as apoptosis, proliferation and differentiation. Looking into their role, miRNAs are an attractive target for cancer therapy. ${ }^{16,17}$ However, involvement of any specific miRNA for its biological functions in GC still remains unclear. A study recently suggested miR-588 was downregulated in lung cancer, and miR-588 showed tumor suppressor activity via regulation of the target gene progranulin. ${ }^{18}$ In a recent study, miR-588 was found to be downregulated in breast cancer. ${ }^{19}$ miR-588 has also been reported to regulate colorectal cancer. ${ }^{20}$ Looking into the reports, miR-588 may function as a tumor suppressor in the progression of cancer; however, the prognostic potential, function and expression pattern of miR-588 have never been studied in human GC.

Invasion and metastasis are associated with EMT, which is evidenced by reduction in levels of E-cadherin and elevation of $\mathrm{N}$-cadherin and vimentin. There are reports suggesting the role of EMT in modulating invasion and metastasis of GC, yet the correlation between EMT and miR-588 in GC is unclear.

In the current investigation, we studied the role of miR588 in the progression of GC. The findings of the present investigation suggested that the expression of miR-588 was decreased significantly in GC-affected tissue specimens. The study found that miR-588 inhibited the invasion and migration of GC cells. The study also found that EIF5A2 is a favorable target of miR-588.

\section{Materials and methods}

\section{Tissue specimens}

About $67 \mathrm{GC}$ tissues along with noncancerous tissues adjacent to them were collected from subjects reported to have GC at The Second Xiangya Hospital, Central South University, Changsha, during the period from March 2015 to March 2017. In accordance with the WHO guidelines, the patients were subjected to pathological diagnosis. Patient history was gathered assuring no chemotherapy and radiation was taken prior to submitting patients to surgery. The experimental protocol received sanction from the ethical committee of The Second Xiangya Hospital, and a signed informed consent was taken from all the patients before the study. The approval number from the human ethical committee was $\mathrm{XCH} / \mathrm{HEC} / 2015 / \mathrm{GC} 14$. The microbiology department of The Second Xiangya Hospital provided all the selected cell lines, which included AGS, MKN-45, BGC-823, MGC-803, SGC7901 along with GES-1 as the normal gastric epithelial cell line. The use of the cell lines was approved by the institutional review board of The Second Xiangya Hospital. The selected cells were seeded in Roswell Park Memorial Institute-1640 medium (Sigma-Aldrich Co., St Louis, MO, USA) which contained $1 \%$ penicillin-streptomycin (Thermo Fisher Scientific, Waltham, MA, USA) and 10\% FBS (Sigma-Aldrich Co.). All the cells were maintained in $5 \% \mathrm{CO}_{2}$ at $37^{\circ} \mathrm{C}$.

\section{Quantitative real-time reverse transcription-PCR (qRT-PCR) studies}

Total RNA was extracted from GC cells and tissues using Trizol reagent (Thermo Fisher Scientific). qRT-PCR study was performed to quantify the expression levels of mature miRNA using NCode miRNA qRT-PCR analysis (Thermo Fisher Scientific). Transcriptional RevertAid First Strand cDNA synthesis Kit (Thermo Fisher Scientific) was used for carrying out reverse transcription reactions. The forward primer of miR-588 was supplied by Thermo Fisher Scientific; GAPDH or the control miRNA U6 primer was selected normalization and was selected as internal loading control for miR-588. The primers were obtained from the primer bank database. qRT-PCR was performed using the 7300 real-time PCR system (Thermo Fisher Scientific).

\section{Determination of expression of proteins by Western blot analysis}

For extracting the proteins from GC cells, RIPA buffer was used. The proteins were isolated on electrophoresis using 5\%-20\% SDS-PAGE gels, followed by transfer to polyvinylidene difluoride membranes. The blocking of membranes was done by $5 \%$ nonfat milk in a mixture of Tris buffer saline and Tween 20, after which the membranes were incubated with antibodies including EIF5A2 $(1: 1,000)$ and the loading control GAPDH $(1: 2,000)$ at $4^{\circ} \mathrm{C}$ for 12 hours. The membranes were exposed to horseradish peroxidase-conjugated II $^{\mathrm{ry}}$ antibody at room temperature for 2 hours. Enhanced chemiluminescence detection reagent (Sigma-Aldrich Co.) was used for the detection and visualization of bands.

\section{Cell migration and invasion assay}

The study of cell migration and invasion was done opting Transwell inserts having Matrigel-coated membranes and a pore size of $8 \mu \mathrm{m}$ (EMD Millipore, Billerica, MA, USA). About $5 \times 10^{4}$ transfected cells were suspended in the upper chamber of Transwell insert. Seven hundred and fifty microliters of medium supplemented with FBS (10\%) was added to 
the lower chamber, which served as the chemoattractant. The Transwell inserts were incubated for 24 hours, following which microscopic counting of cells adhering to the lower surface of membrane was carried out. The cell migration assay was done using a method similar to that followed for the invasion assay, with the difference being the incubation time of 12 hours and no coating of Matrigel to the membranes. The treated GC cells were stained using crystal violet $(0.1 \%)$ for 20 minutes.

\section{Immunohistochemistry}

The $4 \mu \mathrm{m}$ tissue sections were deparaffinized and rehydrated. The sections were subjected to blocking using skim milk $(10 \%)$, retrieving the antigen and suppression of endogenous peroxidase activity. The primary antibodies $\mathrm{N}$-cadherin, vimentin and E-cadherin were obtained from Cell Signaling Technology (Beverly, MA, USA) and the sections were incubated for 12 hours at $4{ }^{\circ} \mathrm{C}$. The peroxidase-conjugated secondary antibody was obtained from Cell Signaling Technology and the sections were incubated for 90 minutes at $4^{\circ} \mathrm{C}$, followed by development using 3,3'-diaminobenzidine solution (Thermo Fisher Scientific) for 5 minutes. The tissue sections were counterstained with hematoxylin dye and were mounted after dehydration.

\section{Evaluation of immunoreactivity}

Immunoreactivity was semi-quantitatively evaluated on the basis of staining intensity and distribution using the immunoreactive score as follows: ${ }^{28,29}$

Immunoreactive score $=$ intensity score $\times$ proportion score

As reported earlier, the intensity score was predefined as follows: 0 , negative; 1 , weak; 2 , moderate and 3 , strong. The proportion score was defined as follows: 0 , negative; $1,<10 \% ; 2,10 \%-50 \% ; 3,50 \%-80 \%$ and $4,>80 \%$ positive cells. After referring the earlier studies, we found that the total score ranged from 0 to 12 .

\section{The miR-588 target prediction analysis}

The prediction of favorable targets was made by downloading TargetDcan predictions for humans, zebrafish and mouse by selecting total context + score and the Probability of Conserved Targeting for prediction scores. We downloaded 3 '-UTR sequences for mouse and humans, followed by splitting each 3'-UTR into 50 nt windows.

\section{The luciferase assay}

The 3'-UTR vector of EIF5A2 was utilized to structure wildtype EIF5A2-3' or the mutant EIF5A2-3'-UTR having the binding sequence for miR-588. The GC cells were subjected to seeding in 24-well plates and were transfected with wild- type and mutant 3 '-UTR and were collected after 24 hours. The luciferase reporter activity was evaluated using Promega dual-luciferase reporter assay system (Promega Corporation, Fitchburg, WI, USA).

\section{In vivo tumor xenografts mice model}

The in vivo metastatic assay was performed by injecting MGC803 cells $\left(1 \times 10^{6}\right.$ cells/ $200 \mu \mathrm{L}$ of PBS $)$ showing decreased expression of miR-588 into nude mice $(n=5)$ via tail vein. Six weeks following the injection of MGC-803 cells, the nude mice were sacrificed and the lungs were dissected for $\mathrm{H} \& \mathrm{E}$ staining. The numbers of metastatic nodules were enumerated using a microscope (Olympus). All the animal protocols received sanction from the ethical committee of The Second Xiangya Hospital, and the experiments were in accordance with the "Animal protection law of the People's Republic of China-2009" for experimental animals. The approval number sanctioned for the study was XCH/AEC/2017/21GC.

\section{Statistical analysis}

Data are presented as mean $\pm \mathrm{SD}$. Comparison of data between groups was done by one-way ANOVA; for multiple comparisons, Fisher's least significant difference method was used. Significance was established at $P<0.05$.

\section{Results}

\section{miR-588 was downregulated in GC tissues and cells}

The levels of miR-588 were estimated in GC tissues and various GC cell lines. All the 67 paired GC tissues and adjacent normal noncancer tissues were subjected to qRT-PCR analysis. Results of the study (Figure 1) showed that the levels of miR588 were significantly downregulated in GC tissues compared to adjacent noncancer tissues. On analyzing miR-588 levels in all the selected GC cell lines, we observed that miR-588 was downregulated in all the cell lines compared to normal GES-1 cells; however, the levels were significantly downregulated in MGC803 cells $(* * P<0.01)$ compared to normal cells. Overall, the outcomes of qRT-PCR suggested downregulation of miR-588 may be associated with the development of GC.

\section{Interrelationship between the levels of miR-588 and prognosis of GC subjects along with poor clinicopathologic features}

To establish the role of miR-588 clinically, we studied the correlation between miR-588 and the clinicopathologic features of $\mathrm{GC}$ in patients included in the study. As shown in Table 1, the mean values of miR-588 were selected as the 

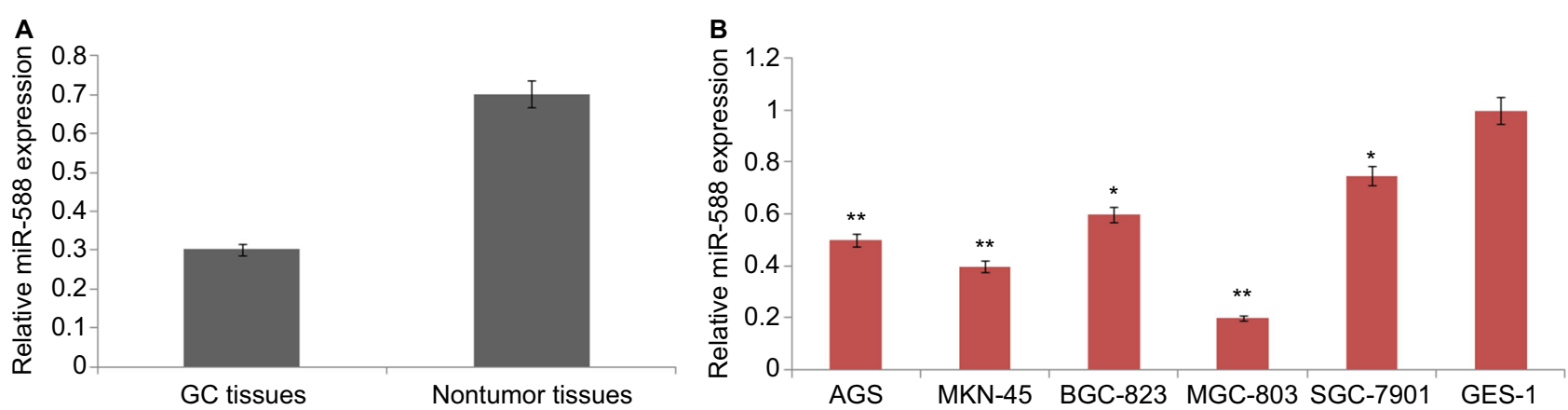

Figure I Expression of miR-588 in selected GC cell lines and tissues.

Notes: (A) The mean levels of miR-588 in GC tissues were significantly on the lower side compared to adjacent nontumor tissues (*P<0.0I compared to noncancer tissues). (B) The expression of mean miR-588 levels in different GC and GES-I cell lines ( ${ }^{*} P<0.05,{ }^{* * P}<0.01$ compared to control GES-I cells).

Abbreviation: GC, gastric cancer.

Table I The parameters correlating the expression of miR-588 and associated clinicopathologic features observed in GC patients $(\mathrm{N}=67)$

\begin{tabular}{|c|c|c|c|c|}
\hline \multirow[t]{2}{*}{ Clinical parameters } & \multirow[t]{2}{*}{ Number of cases } & \multicolumn{2}{|l|}{ Level of expression } & \multirow[t]{2}{*}{$P$-value } \\
\hline & & High miR-588 (3I) & Low miR-588 (36) & \\
\hline \multicolumn{5}{|l|}{ Gender } \\
\hline Male & 46 & 21 & 25 & $0.75 \mathrm{I}$ \\
\hline Female & 21 & 10 & 11 & \\
\hline \multicolumn{5}{|l|}{ Age of patients (years) } \\
\hline$<60$ & 24 & 11 & 14 & 0.625 \\
\hline$\geq 60$ & 43 & 20 & 22 & \\
\hline \multicolumn{5}{|l|}{ Size of tumor $(\mathrm{cm})$} \\
\hline$<5$ & 53 & 25 & 27 & $0.50 \mathrm{I}$ \\
\hline$\geq 5$ & 14 & 6 & 9 & \\
\hline \multicolumn{5}{|l|}{ Type } \\
\hline Intestinal & 54 & 19 & 30 & 0.312 \\
\hline Diffuse & 13 & 12 & 6 & \\
\hline \multicolumn{5}{|l|}{ TNM stage } \\
\hline $\mathrm{I}+\mathrm{II}$ & 17 & 10 & 5 & $0.005^{*}$ \\
\hline III+IV & 50 & 21 & 31 & \\
\hline \multicolumn{5}{|l|}{ Lymph node metastasis } \\
\hline Present & 48 & 17 & 30 & $0.002 *$ \\
\hline Absent & 19 & 14 & 6 & \\
\hline \multicolumn{5}{|l|}{ Metastasis type } \\
\hline Distant metastasis & 09 & 03 & 06 & $0.008^{*}$ \\
\hline Nondistant metastasis & 58 & 28 & 30 & \\
\hline
\end{tabular}

Note: ${ }^{*} P<0.05$.

Abbreviation: GC, gastric cancer.

demarcation point. The results showed that downregulation of miR-588 was the main feature of lymph node metastasis and in advanced TNM stage. We found that the expression level of miR-588 was a feature for both disease-free survival and 5-year overall survival in patients reported as having GC (Table 2). Hence, the outcomes show miR-588 as a potential biomarker in the development and progression of GC.

\section{miR-588 inhibits the invasion and migration of MGC803 GC cells in vitro and in vivo}

To investigate the role of miR-588 in GC cells, we transfected the MGC803 GC cells with miR-588 vector or
anti-miR-588 vector. qRT-PCR was done to evaluate the expression levels of miR-588, and the results of expression are depicted in Figure 2. The results suggest that miR-588 was overexpressed significantly in MGC803 cells (Figure 2A) and suppressed in SGC7901 cells $\left({ }^{*} P<0.05\right.$, $* * P<0.01$; Figure $2 \mathrm{~B}, \mathrm{C}$ ). The migration and invasion assay was done by Matrigel transwell assay (coated and uncoated). The overexpression of miR-588 halted the migration and invasiveness in MGC 803 cells $\left({ }^{*} P<0.05\right.$; Figure $\left.2 \mathrm{~B}\right)$. However, the number of SGC7901 cells reported to be invaded and migrated increased ( $* * P<0.01$; Figure $2 \mathrm{D})$; also, the expression levels of miR-588 were found to be decreased (Figure 2D) in these cells. 
Table 2 Five-year Cox regression analysis (multivariate) of OS and DFS in 67 patients identified with GC

\begin{tabular}{|l|l|l|l|l|l|l|}
\hline \multirow{2}{*}{ Variables } & \multicolumn{2}{l}{ OS } & \multicolumn{2}{l|}{ DFS } \\
\cline { 2 - 7 } & Hazard ratio & $\mathbf{9 5 \%} \mathbf{~ C l}$ & P-value & Hazard ratio & $\mathbf{9 5 \%}$ Cl & P-value \\
\hline Expression of miR-588 & 0.187 & $0.022-0.922$ & $0.008^{*}$ & 0.220 & $0.060-0.860$ & $0.015^{*}$ \\
\hline Lymph node metastasis & 3.056 & $1.32-5.2 I$ & $0.004^{*}$ & 3.010 & $1.35 I-5.423$ & $0.005^{*}$ \\
\hline Stage & & & & & & \\
\hline T stage & 1.52 & $1.052-2.48 I$ & $0.007^{*}$ & 1.87 & $0.913-3.033$ & $0.004^{*}$ \\
\hline N stage & 2.89 & $1.982-5.452$ & $0.002^{*}$ & 3.05 & $1.4 I I-6.226$ & $0.003^{*}$ \\
\hline M stage & 3.98 & $2.32 I-7.562$ & $0.00 I^{*}$ & 4.07 & $0.89 I-8.45$ & $0.00 I^{*}$ \\
\hline
\end{tabular}

Note: *Statistically significant.

Abbreviations: DFS, disease-free survival; GC, gastric cancer; OS, overall survival.

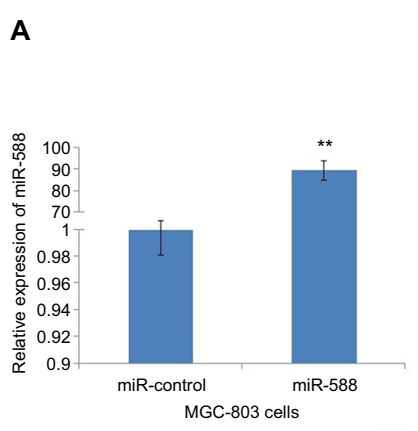

C

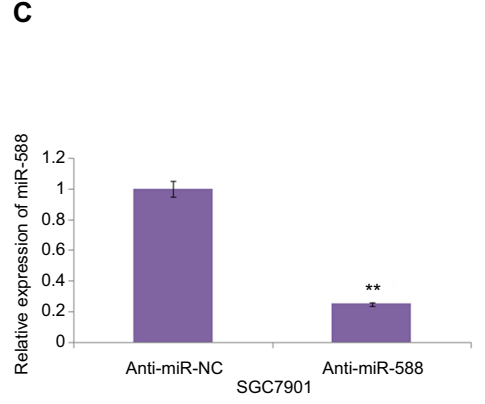

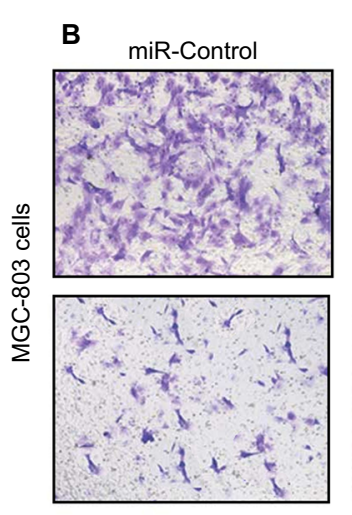
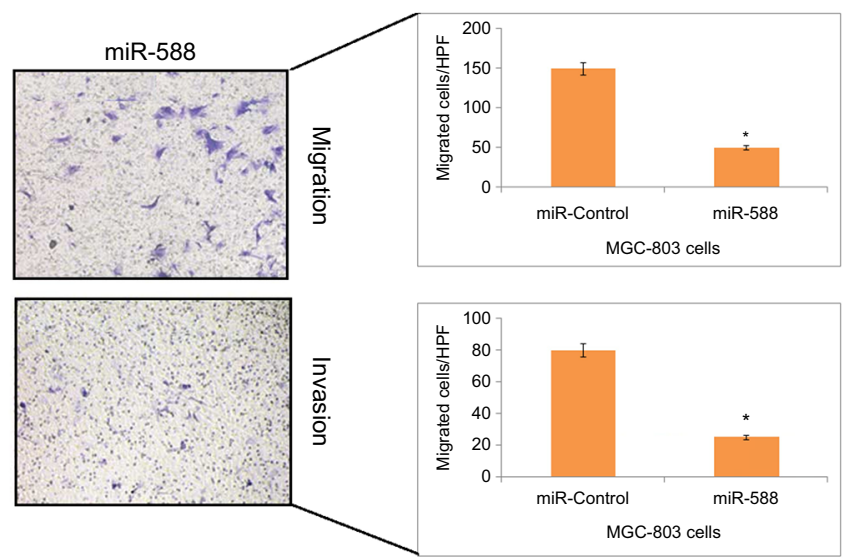

D
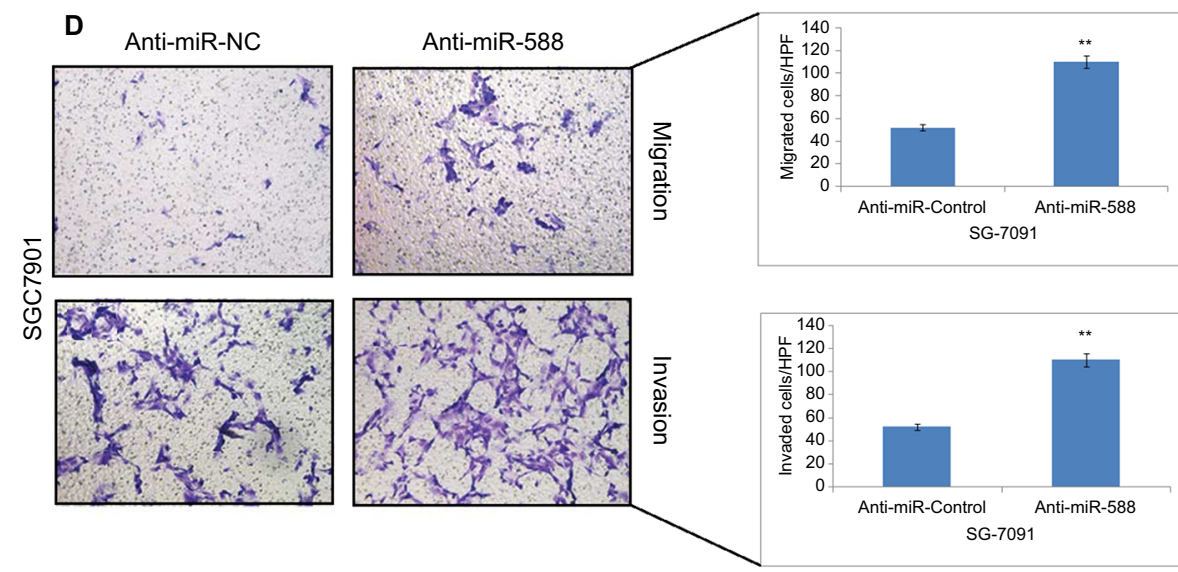

Figure 2 miR-588 exerted inhibitory effect on the invasion and migration of GC cells in vitro.

Notes: (A) qRT-PCR study for the expression of miR-588 in MGC-803 cells followed by transfection using specific miRNA vectors (**P<0.01 compared to miR-control). (B) Cell migration and invasion by transwell chamber assay. Overexpression of miR-588 exerted an inhibitory effect on MGC-803 cells ( ${ }^{*}<0.05$ compared to miR-control). (C) qRT-PCR analysis for the expression of miR-588 was done in SGC-790I cells after transfecting them with anti-miR-588 and negative control (**P<0.0I compared to anti-miR-NC). (D) Transwell chamber assay for the study of migration and invasion of SGC-790I cells with knocked out miR-588. The extent of cell migration and invasion was distinctly improved in SGC-790I cells (**P<0.0I compared to anti-miR-NC).

Abbreviations: GC, gastric cancer; qRT-PCR, quantitative real-time reverse transcription-PCR; HPF, high power field.

Mice model of GC having lung metastasis was developed in order to investigate the role of miR-588 in vivo. The mice were injected with MGC-803 cells via tail vein. The nude mice were then injected with lentiviral constructs, that is, LV-miR-588 or LV-control MGC803 cells and LV-anti-miR-588 or LV-anti-miR-
$\mathrm{NC}$, through the tail vein. The results suggested that overexpression of miR-588 suppressed the lung metastasis of MGC803 cells, whereas suppression of miR-588 enhanced the extent of pulmonary metastasis in nude mice $(* P<0.05$; Figure 3$)$. The outcome of experiment supported the role of miR-588 on GC 


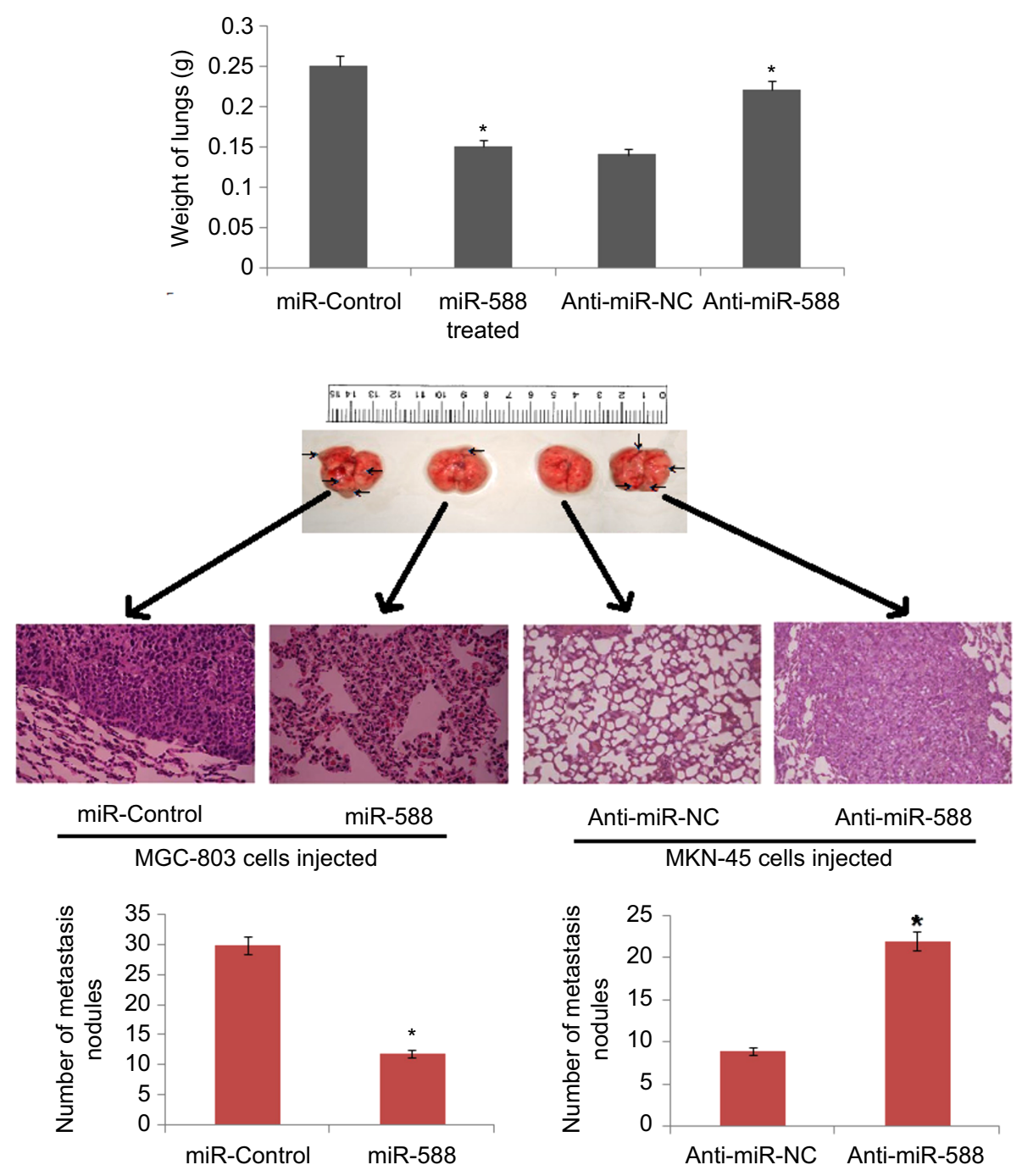

Figure 3 The photographs show dissected lungs, the arrows show the presence of nodules in lungs and the scale bar shows the size.

Notes: The weight of lungs dissected was assessed and analyzed statistically. H\&E staining of lung metastasis of MGC803 or SGC-790I cells after transfection with the respective miR-588 vector. ${ }^{*} P<0.05$ compared to miR-control, ${ }^{*} P<0.05$ compared to anti-miR-NC.

cells and confirmed that miR-588 has an important role in the invasion and migration of GC cells both in vitro and in vivo.

\section{miR-588 inhibits EMT in GC cells}

EMT has been identified to play an important role in the development and progression of metastasis in cancer. To study the involvement of miR-588 in GC metastasis, important markers of EMT were evaluated. The outcomes of the experiment suggested that overexpression of miR-588 resulted in suppression of vimentin and $\mathrm{N}$-cadherin, while the levels of E-cadherin were elevated $(* P<0.05$; Figure $4 \mathrm{~A})$. The downregulation of miR-588 reversed the effects as the levels of vimentin and $\mathrm{N}$-cadherin were elevated, whereas that of E-cadherin was suppressed ( ${ }^{*} P<0.05$; Figure $4 \mathrm{~A}$ ).

\section{Effect of miR-588 expression on the expression of E-cadherin and vimentin in GC tissues}

Expression of E-cadherin and vimentin in GC tissues with high and low expression level of miR-588 is presented in Table 3 . We found that E-cadherin was highly expressed in the group showing high expression of miR-588 compared to that showing lower miR-588 expression. The results were opposite with respect to vimentin, as the levels were toward significantly higher side in the group showing lower expression of miR-588 compared to that showing higher expression of miR-588 (Figure 4B). The experiment established the suppressive effect of miR-588 for EMT in GC cells. 


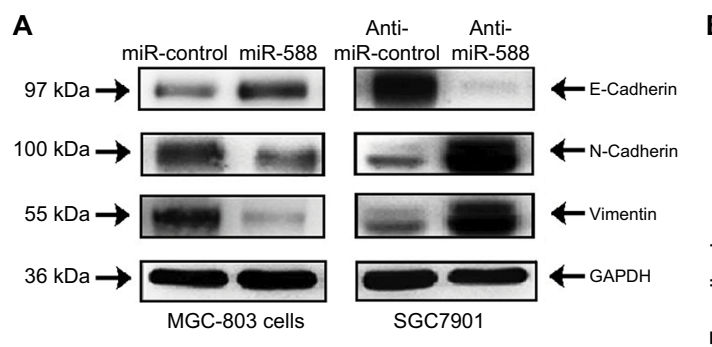

B
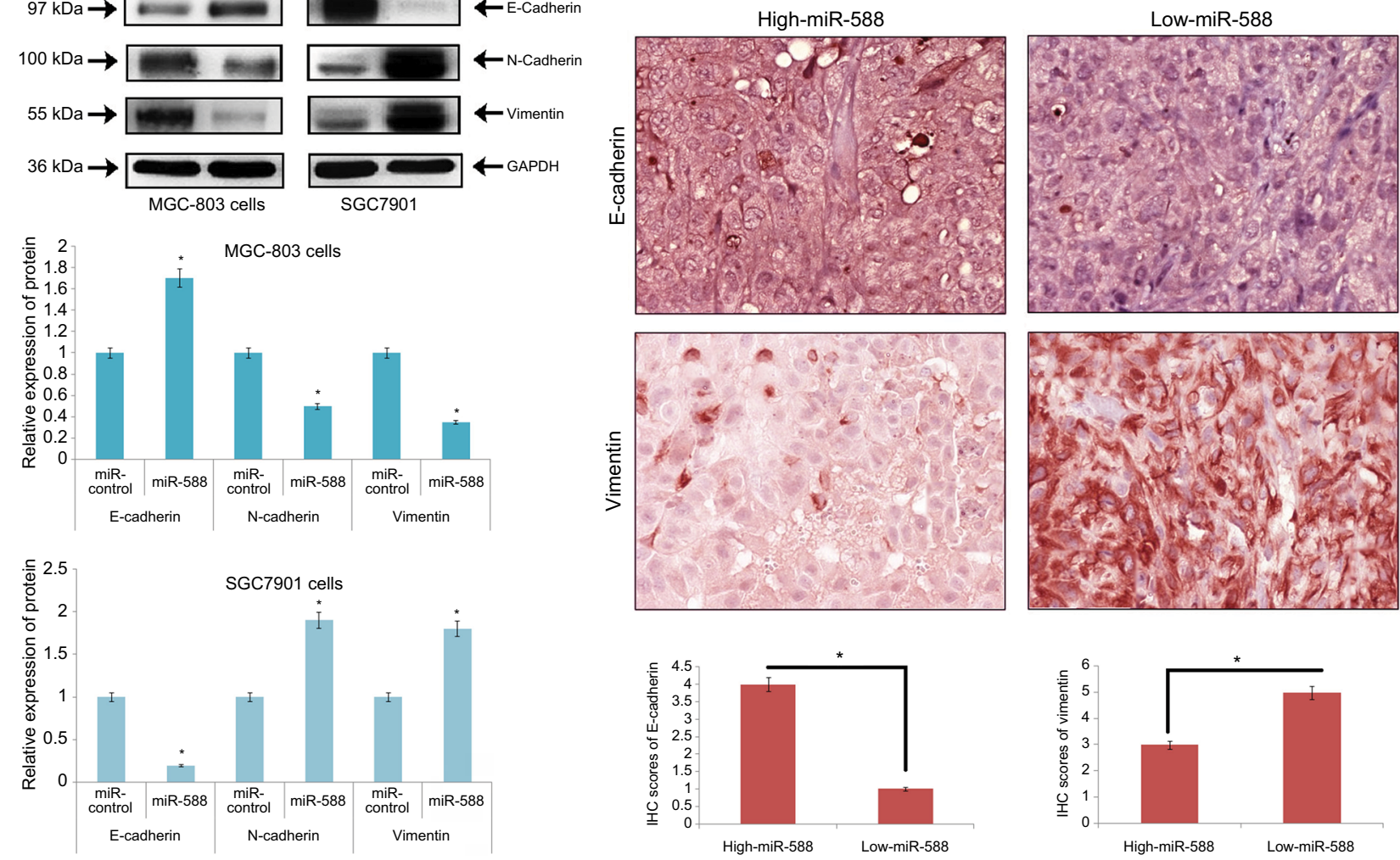

Figure 4 miR-588 suppressed EMT in GC cells.

Notes: (A) Western blot studies for the expression of proteins. E-cadherin was overexpressed, whereas the levels of $\mathrm{N}$-cadherin and vimentin were suppressed in miR588transfected MGC-803 cells. In SGC-790I cells, transfection with anti-miR-588 resulted in suppression of E-cadherin and upregulation of both N-cadherin and vimentin $(* P<0.05$ compared to miR-control). (B) IHC analysis of GC tissue samples for the expression of E-cadherin and vimentin. The IHC score of E-cadherin was significantly on the lower side, whereas that of vimentin was on the higher side in low miR-588 expressing GC tissues. (Values are mean $\pm S D$; $* P<0.05$ compared to high miR-588 tissues.) Abbreviations: EMT, epithelial-mesenchymal transition; GC, gastric cancer; IHC, immunohistochemical.

Table 3 Comparison between high and low miR-588 expressing GC tissues for E-cadherin and vimentin expression

\begin{tabular}{|l|l|l|l|l|l|l|}
\hline \multirow{2}{*}{ Groups } & \multicolumn{2}{|l|}{ E-cadherin } & \multirow{2}{*}{ P-value } & \multicolumn{2}{|l|}{ Vimentin } & \multirow{2}{*}{ P-value } \\
\cline { 2 - 5 } & $\leq \mathbf{4}$ & $\mathbf{5 - 1 2}$ & & $\leq \mathbf{4}$ & $\mathbf{5 - 1 2}$ & \\
\hline $\begin{array}{l}\text { High miR-588 } \\
\text { expressing }(\mathrm{n}=20)\end{array}$ & 7 & 13 & 0.05 & 17 & 3 & 0.360 \\
\hline $\begin{array}{l}\text { Low miR-588 } \\
\text { expressing }(\mathrm{n}=47)\end{array}$ & 8 & 39 & 0.01 & 37 & 10 & 0.05 \\
\hline
\end{tabular}

Abbreviation: GC, gastric cancer.

\section{EIF5A2 was the potential target of miR- 588 in GC cells}

In the line to identify the direct target of miR-588 in silico, algorithm TargetScan was used for identifying the possible mechanism by which miR-588 acts on the migration and invasion capacity of GC cells. The algorithm TargetScan suggested sequence match between miR-588 and 3'-UTR of EIF5A2. After this, EIF5A2 was evaluated by luciferase assay for being a direct target of miR-588 in GC cells. The luciferase activity of the wild-type (WT)-EIFA2-3'-UTR was suppressed by overexpression of miR-588, whereas that of mutant type (MT)-EIF5A2-3'-UTR remained unchanged $(* P<0.05$; Figure $5 \mathrm{~A})$. In addition, the luciferase activity of MT-EIF5A2-3'-UTR constructs remained unaffected when miR-588 was suppressed. On the contrary, the luciferase activity improved $(* P<0.05$; Figure $5 \mathrm{~A})$ in WT-EIF5A2-3'-UTR. The results also demonstrated that overexpression of miR-588 caused suppression of protein EIF5A 2 and mRNA in GC cells ( ${ }^{*} P<0.05$; Figure $\left.5 \mathrm{~B}, \mathrm{C}\right)$; however, inhibiting miR-588 resulted in overexpression of both mRNA and protein expression of EIA5A2 in MKN-45 cells $\left({ }^{*} P<0.05\right)$.

\section{High miR-588 expression suppressed EIF5A2 mRNA expression in GC tissues}

To study the effect of miR-588 on the expression of mRNA and protein levels of EIF5A2 in vivo, the expression levels of EIF5A2 mRNA were evaluated (Figure 6A) in GC 

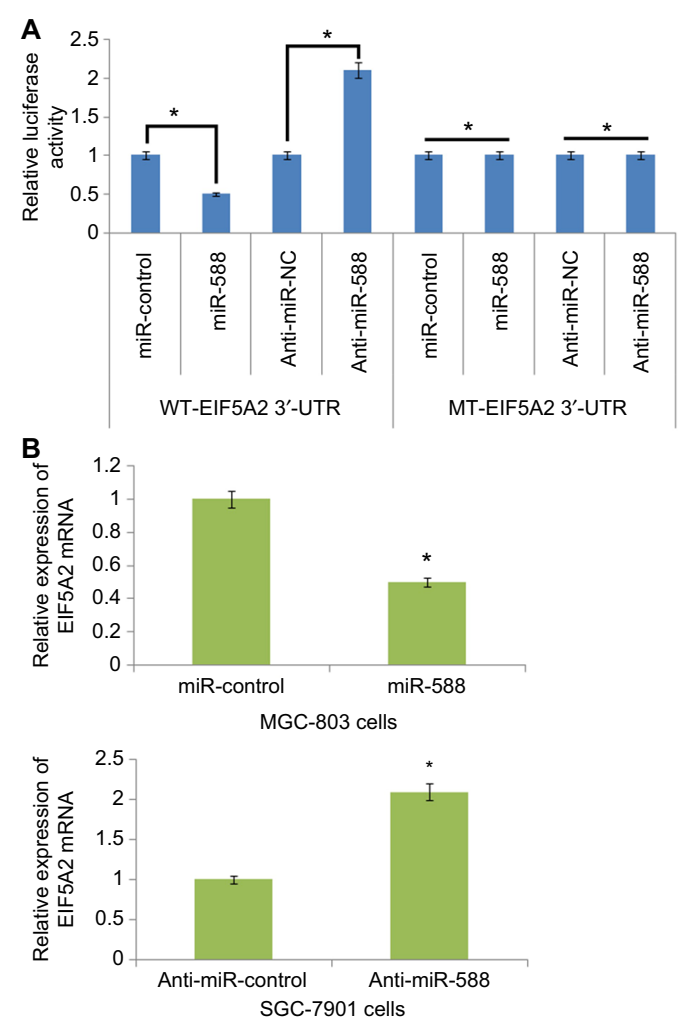

C
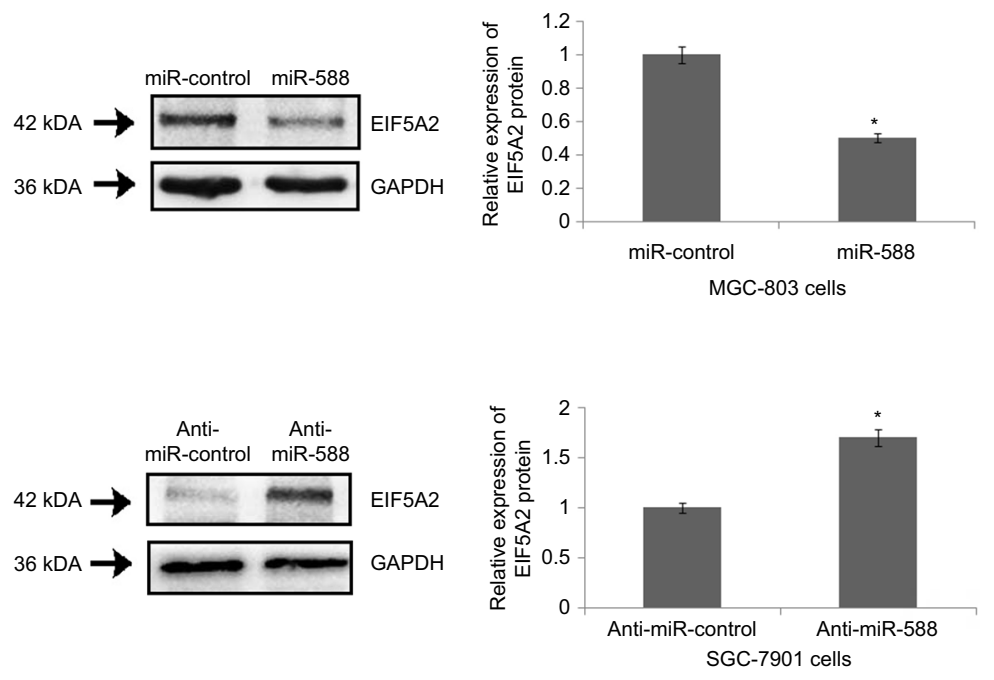

Figure 5 EIF5A2 is a direct target of miR-588 in gastric cancer.

Notes: (A) The WT-3'-UTR-EIF5A2 altered luciferase activity. The luciferase activity of WT-3'-UTR-ElF5A2 was significantly enhanced by anti-miR-588 compared to negative control (anti-miR-NC), whereas the MT produced no effects on the levels of luciferase activity. (B) qRT-PCR analysis for the expression of EIF5A2 mRNA in miR588-transfected MGC-803 and SGC-790I cells. (C) Expression of proteins by Western blot suggested that in MGC-803 cells, the expression of EIF5A2 was suppressed after transfection with miR-588, and the levels were elevated in SGC-790I cells after transfection with anti-miR-588 compared to respective controls $(* P<0.05$ compared to control).

Abbreviations: MT, mutant type; qRT-PCR, quantitative real-time reverse transcription-PCR; WT, wild type.

tissues. The outcomes of the study suggested that the GC tissues showed higher expression of miR-588 with lower levels of EIF5A2 mRNA compared to adjacent normal tissues which exhibited lower miR-588 levels and higher expressing levels of EIF5A2 cancer tissues $\left({ }^{*} P<0.05\right.$; Figure $6 \mathrm{~B}$ ). The study clearly suggested that miR-588 influenced the levels of EIF5A2 in vivo in the tissues $(r=-0.7288, * P<0.05$; Figure $6 \mathrm{C})$. The outcomes suggested that EIF5A2 was the downstream target of miR-588 in $\mathrm{GC}$ in vivo.

\section{EIF5A2 acts as a downstream mediator in miR-588-mediated cell invasion, migration and progression of EMT in GC}

For affirming EIF5A2 as a potential target of miR-588 in GC, we studied the role of EIF5A2 in miR-588-arbitrated cell invasion, migration and EMT progression in GC. Upon transfecting the cells with EIF5A2-expressing plasmid, the expression of EIF5A2 was retrieved in MGC803-miR588 cells and the relative expression of protein EIF5A2 was significantly higher compared to cells transfected with the empty vector $\left({ }^{*} P<0.05\right.$; Figure $\left.7 \mathrm{~A}\right)$. The MGC-803 cells showed overexpression of miR-588, and the process of cell invasion and migration increased significantly, suggesting EIF5A2 influenced cell migration, invasion and EMT progression of GC cells $\left({ }^{*} P<0.05\right.$; Figure $\left.7 \mathrm{~B}\right)$. The miR-588-suppressive SGC-7901 cells were knocked out for EIF5A2 by a specific siRNA. The results suggest the inhibitory effect on cell invasion and migration (Figure 7C, D). The progression of EMT was marked by EMT marker proteins in MGC-803 cells (Figure 7E) and transfected SGC-7901 cells (Figure 7F). Overall, the outcomes suggested that EIF5A2 is a downstream target acting on miR-588 in GC.

\section{Discussion}

MicroRNAs, also called as miRNAs, are identified as an important group of noncoding RNAs involved in the development and progression of cancers..$^{21,22}$ Number of studies 


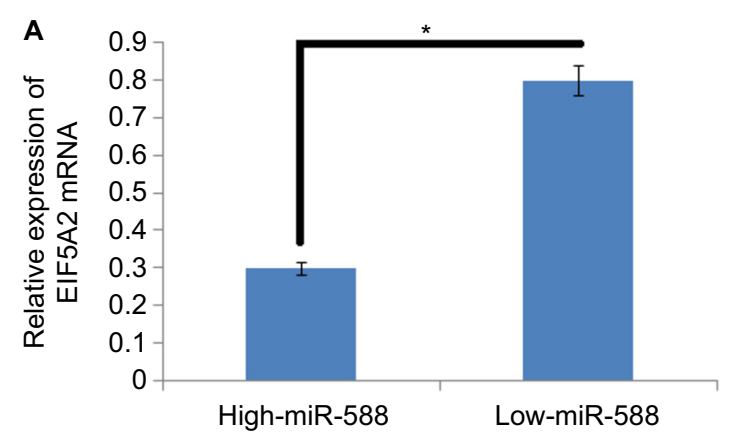

High-miR-588

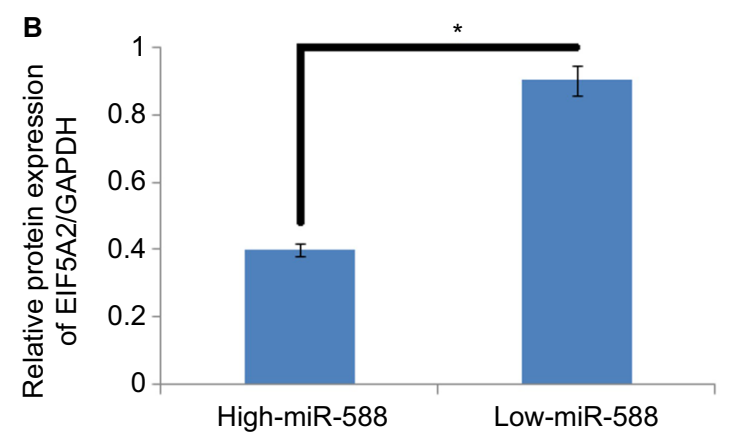

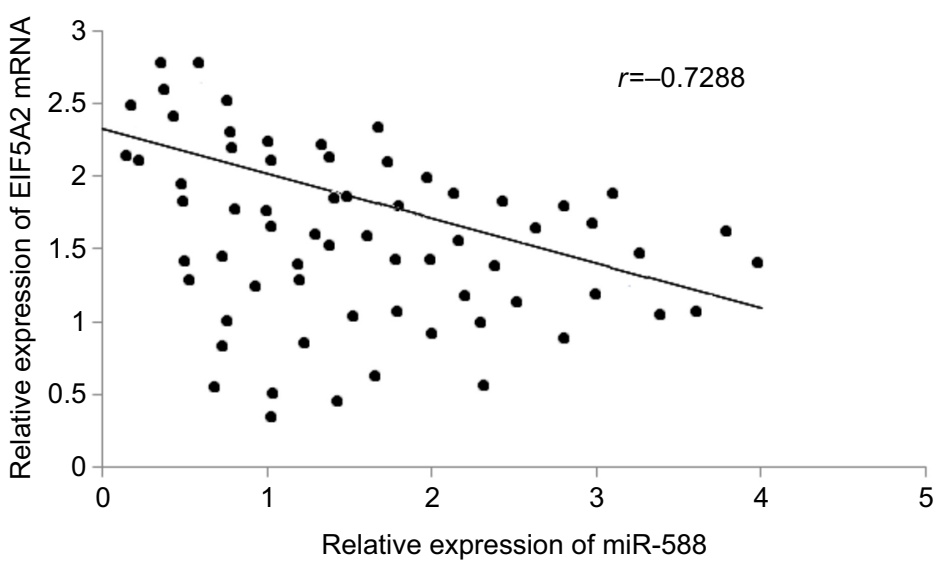

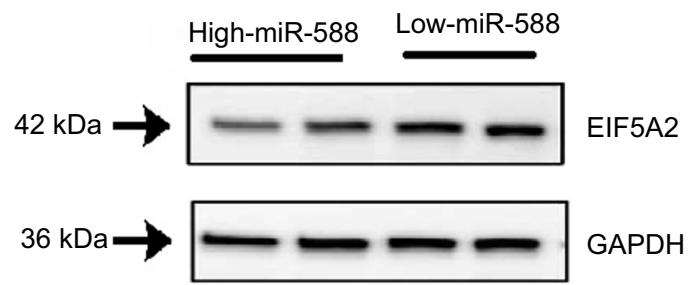

Figure 6 An inverse relationship was observed between the expression of miR-588 and levels of EIF5A2 in GC tissues.

Notes: (A) The mRNA expression levels of EIF5A2 were significantly on the lower side in high-expression group of miR-588 compared to that in the low-expression group $(* P<0.05)$. (B) The levels of protein ElF5A2 were suppressed in high miR-588 group compared to the low miR-588 group. (C) An inverse correlation existed between the mRNA levels of EIF5A2 and miR-588 in GC tissue samples included in the study $(* P<0.05)$.

Abbreviation: GC, gastric cancer.

have identified miRNAs as important therapeutic targets in treating GC. ${ }^{23,24}$ Literatures have suggested miR-588 is downregulated in lung cancer and breast cancer; ${ }^{18,19}$ miR-588 is also reported to regulate colorectal cancer. ${ }^{20}$ The results of our study suggested that downregulation of miR-588 was associated with lymph node metastasis and advanced TNM stage in GC. For overall and disease free survival predicted for 5 years, expression of miR-588 served as indicator. The study also affirmed that miR-588 may play an important role in the development and progression of GC; also, suppression of miR-588 marked poor prognosis, establishing miR-588 as a prognostic marker for GC.

An earlier study has reported that miR-588 plays an important role in cell invasion; ${ }^{18}$ however, its role as an oncogene or a tumor suppressor varies with the type of cancer. In the present study, in vivo and in vitro experiments were done to find the role of miR-588 in cell invasion, migration and progression of EMT. The outcomes clarified that miR-588 suppressed cell invasion, migration and progression of EMT. EIF5A2 is identified to be a potential oncogenic protein in human cancer, ${ }^{25,26}$ which is overexpressed in GC, promoting metastasis and growth in cancer, thus establishing itself as a forecaster for poor survival. ${ }^{24,27}$ The results of luciferase assay confirmed EIF5A2 as a downstream target of miR-588. We also found that miR-588 controlled the expression of EIF5A2 negatively in GC cells. Our results indicated that the inhibitory effect of miR-588 on the invasion and migration of GC cells was abolished by overexpression of EIF5A2. We also found that suppression of EIF5A2 improved the miR588-mediated invasion and migration of GC cells. Hence, these outcomes confirmed that EIF5A2 acted as a mediator of miR-588 in GC.

In the study, we confirmed that miR-588 prevented cell inhibition, migration and progression of EMT via suppressing EIF5A2. Overall, miR-588 acted as a tumor suppressor in GC and could be a potential target in treating GC. 
A

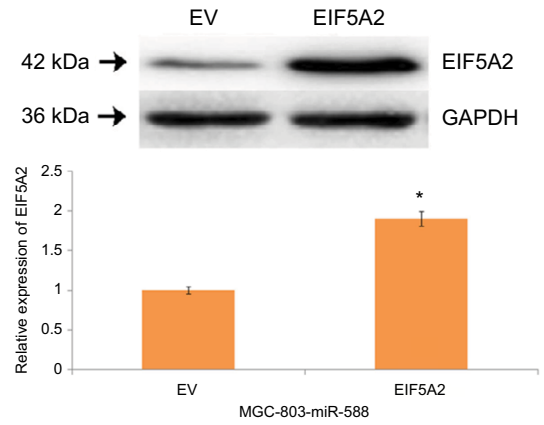

C Scrambled siRNA EIF5A2-siRNA
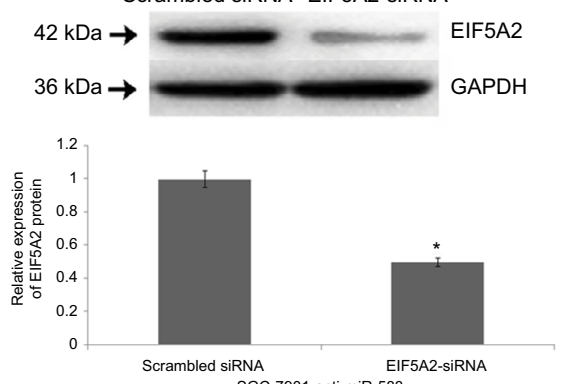

SGC-7901-anti-miR-588
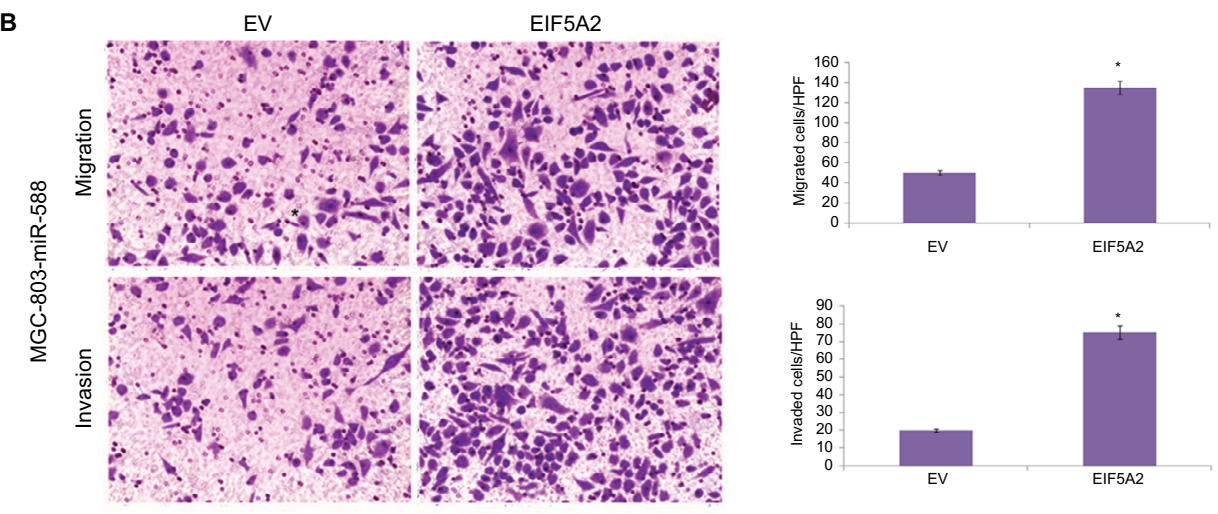

D
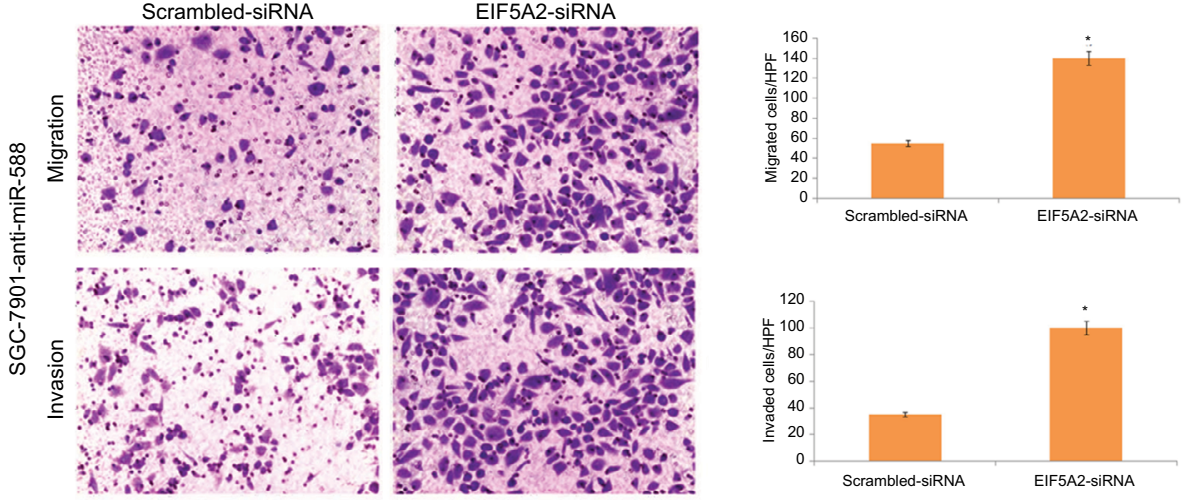

E
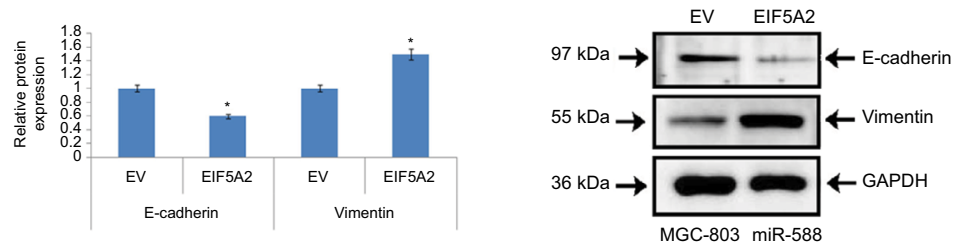

$\mathbf{F}$
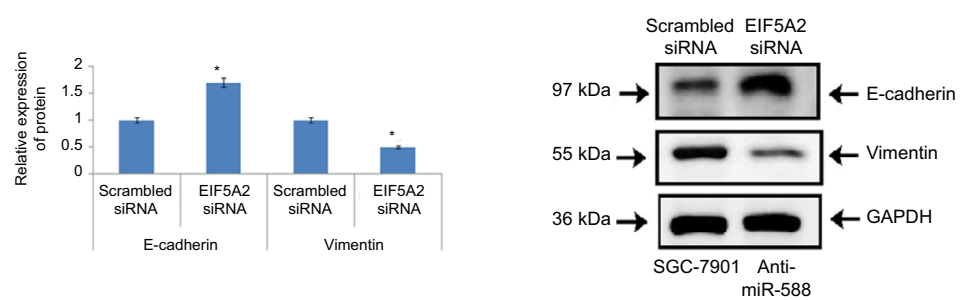

Figure 7 miR-588-influenced migration, invasion and EMT were partially blocked by causing alterations in levels of EIF5A2.

Notes: (A) Western blot analysis for the expression of EIF5A2 in miR-588-overexpressing MGC803 cells followed by transfection with EV or EIF5A2 expression plasmid $(* P<0.05$ compared to EV). (B) In MGC-803 cells exhibiting overexpression of miR-588, overexpression of ElF5A2 increased the process of migration and invasion in cells (*P<0.05 compared to EV). (C) The SGC-790I cells exhibiting lower miR-588 levels were transfected with scrambled siRNA or ElF5A2 siRNA, following which Western blot analysis was done for the expression of EIF5A2 ( $* P<0.05$ compared to scrambled siRNA). (D) EF5A2 was knocked out in SGC-790I cells, which was followed by recording the effects of miR-588 knockdown ( $* P<0.05$ compared to scrambled siRNA). (E) The markers of EMT were studied in transfected MGC-803 and (F) SGC-790I cells by Western blot analysis $(* P<0.05$ compared to scrambled siRNA).

Abbreviations: EMT, epithelial-mesenchymal transition; EV, empty vector; HPF, high power field. 


\section{Acknowledgments}

The authors are thankful to the National Natural Science Foundation of China for providing financial grant for the project. The authors are also thankful to the staff and management of Department of Gerontology, The Second Xiangya Hospital, Central South University, China, for providing the necessary facilities.

This work is supported by grants from the National Natural Science Foundation of China (No. 81500662).

\section{Disclosure}

The authors report no conflicts of interest in this work.

\section{References}

1. Siegel R, Naishadham D, Jemal A. Cancer statistics, 2013. CA Cancer J Clin. 2013;63(1):11-30.

2. Kamangar F, Dores GM, Anderson WF. Patterns of cancer incidence, mortality, and prevalence across five continents: defining priorities to reduce cancer disparities in different geographic regions of the world. J Clin Oncol. 2006;24(14):2137-2150.

3. Valastyan S, Weinberg RA. Tumor metastasis: molecular insights and evolving paradigms. Cell. 2011;147(2):275-292.

4. Kang Y, Massagué J. Epithelial-mesenchymal transitions: twist in development and metastasis. Cell. 2004;118(3):277-279.

5. Orditura M, Galizia G, Sforza V, et al. Treatment of gastric cancer. World J Gastroenterol. 2014;20(7):1635-1649.

6. Thrumurthy SG, Chaudry MA, Chau I, Allum W. Does surgery have a role in managing incurable gastric cancer? Nat Rev Clin Oncol. 2015;12(11):676-682.

7. Ying SY, Chang DC, Lin SL. The microRNA (miRNA): overview of the RNA genes that modulate gene function. Mol Biotechnol. 2008;38(3):257-268.

8. Guo H, Ingolia NT, Weissman JS, Bartel DP. Mammalian microRNAs predominantly act to decrease target mRNA levels. Nature. 2010;466(7308):835-840.

9. Shukla GC, Singh J, Barik S. MicroRNAs: processing, maturation, target recognition and regulatory functions. Mol Cell Pharmacol. 2011;3(3):83-92.

10. Jiang C, Chen X, Alattar M, Wei J, Liu H. MicroRNAs in tumorigenesis, metastasis, diagnosis and prognosis of gastric cancer. Cancer Gene Ther. 2015;22(6):291-301.

11. Lu J, Getz G, Miska EA, et al. MicroRNA expression profiles classify human cancers. Nature. 2005;435(7043):834-838.

12. Mcmanus MT. MicroRNAs and cancer. Semin Cancer Biol. 2003;13(4): 253-258.

13. Korourian A, Roudi R, Shariftabrizi A, Madjd Z. MicroRNA-31 inhibits RhoA-mediated tumor invasion and chemotherapy resistance in MKN-45 gastric adenocarcinoma cells. Exp Biol Med. 2017;242(18):1842-1847.
14. Ma M, Chen S, Liu Z, et al. miRNA-221 of exosomes originating from bone marrow mesenchymal stem cells promotes oncogenic activity in gastric cancer. Onco Targets Ther. 2017;10:4161-4171.

15. Yue H, Tang B, Zhao Y, et al. MIR-519d suppresses the gastric cancer epithelial-mesenchymal transition via Twist1 and inhibits Wnt/ $\beta$-catenin signaling pathway. Am J Transl Res. 2017;9(8): 3654-3664.

16. Calin GA, Croce CM. MicroRNA-cancer connection: the beginning of a new tale. Cancer Res. 2006;66(15):7390-7394.

17. Shrestha S, Hsu SD, Huang WY, et al. A systematic review of microRNA expression profiling studies in human gastric cancer. Cancer Med. 2014;3(4):878-888.

18. Qian L, Lin L, Du Y, Hao X, Zhao Y, Liu X. MicroRNA-588 suppresses tumor cell migration and invasion by targeting GRN in lung squamous cell carcinoma. Mol Med Rep. 2016;14(4):3021-3028.

19. Yu M, Zhang X, Li H, Zhang P, Dong W. MicroRNA-588 is downregulated and may have prognostic and functional roles in human breast cancer. Med Sci Monit. 2017;23:5690-5696.

20. Kohlhapp FJ, Mitra AK, Lengyel E, Peter ME. MicroRNAs as mediators and communicators between cancer cells and the tumor microenvironment. Oncogene. 2015;34(48):5857-5868.

21. Liu Z, Dou C, Yao B, et al. Ftx non coding RNA-derived miR-545 promotes cell proliferation by targeting RIG-I in hepatocellular carcinoma. Oncotarget. 2016;7(18):25350-25365.

22. Liu Z, Dou C, Yao B, et al. Methylation-mediated repression of microRNA-129-2 suppresses cell aggressiveness by inhibiting high mobility group box 1 in human hepatocellular carcinoma. Oncotarget. 2016;7(24):36909-36923.

23. Rao M, Zhu Y, Zhou Y, Cong X, Feng L. MicroRNA-122 inhibits proliferation and invasion in gastric cancer by targeting CREB1. Am J Cancer Res. 2017;7(2):323-333.

24. Tian SB, Yu JC, Liu YQ, et al. MiR-30b suppresses tumor migration and invasion by targeting EIF5A2 in gastric cancer. World J Gastroenterol. 2015;21(31):9337-9347.

25. Huang PY, Zeng TT, Ban X, et al. Expression of EIF5A2 associates with poor survival of nasopharyngeal carcinoma patients treated with induction chemotherapy. BMC Cancer. 2016;16:669.

26. Deng B, Wang B, Fang J, et al. MiRNA-203 suppresses cell proliferation, migration and invasion in colorectal cancer via targeting of EIF5A2. Sci Rep. 2016;6:28301.

27. Yang SS, Gao Y, Wang DY, et al. Overexpression of eukaryotic initiation factor 5A2 (EIF5A2) is associated with cancer progression and poor prognosis in patients with early-stage cervical cancer. Histopathology. 2016;69(2):276-287.

28. Chui X, Egami H, Yamashita J, et al. Immunohistochemical expression of the c-kit proto-oncogene product in human malignant and non-malignant breast tissues. Br J Cancer. 1996;73(10): 1233-1236.

29. Engels K, Knauer SK, Metzler D, et al. Dynamic intracellular survivin in oral squamous cell carcinoma: underlying molecular mechanism and potential as an early prognostic marker. J Pathol. 2007;211(5): $532-540$.
Cancer Management and Research

\section{Publish your work in this journal}

Cancer Management and Research is an international, peer-reviewed open access journal focusing on cancer research and the optimal use of preventative and integrated treatment interventions to achieve improved outcomes, enhanced survival and quality of life for the cancer patient. The manuscript management system is completely online and includes
Dovepress

a very quick and fair peer-review system, which is all easy to use. Visit $\mathrm{http}: / / \mathrm{www}$.dovepress.com/testimonials.php to read real quotes from published authors. 